Service social

\title{
Les facteurs de risque de la prostitution des jeunes filles mineures à Bobo-Dioulasso au Burkina Faso
}

\section{Yvonne Clémence Bambara}

Volume 58, numéro 1, 2012

URI : https://id.erudit.org/iderudit/1010445ar

DOI : https://doi.org/10.7202/1010445ar

Aller au sommaire du numéro

Éditeur(s)

École de service social de l’Université Laval

ISSN

1708-1734 (numérique)

Découvrir la revue

Citer ce document

Bambara, Y. C. (2012). Les facteurs de risque de la prostitution des jeunes filles mineures à Bobo-Dioulasso au Burkina Faso. Service social, 58(1), 110-124. https://doi.org/10.7202/1010445ar
Résumé de l'article

L'objectif de la recherche était d'identifier d'une part les caractéristiques personnelles, familiales, sociales et culturelles des jeunes filles mineures en situation de prostitution à Bobo-Dioulasso, et d'autre part, les motifs les y ayant conduites. Un guide d'entretien semi-structuré a été administré individuellement à 15 jeunes filles de 14 à 18 ans. L'analyse qualitative des verbatim révèle que plusieurs facteurs de risque ont exposé les jeunes filles enquêtées à la prostitution à savoir : la violence et les maltraitances subies dans le giron familial, les abus sexuels précoces, l'absence d'instruction et de formation professionnelle et la précarité économique des parents. 


\title{
Les facteurs de risque de la prostitution des jeunes filles mineures à Bobo-Dioulasso au Burkina Faso*
}

Yvonne Clémence BAMBARA

\begin{abstract}
RÉSUMÉ
L'objectif de la recherche était d'identifier d'une part les caractéristiques personnelles, familiales, sociales et culturelles des jeunes filles mineures en situation de prostitution à BoboDioulasso, et d'autre part, les motifs les y ayant conduites. Un guide d'entretien semi-structuré a été administré individuellement à 15 jeunes filles de 14 à 18 ans. L'analyse qualitative des verbatim révèle que plusieurs facteurs de risque ont exposé les jeunes filles enquêtées à la prostitution à savoir : la violence et les maltraitances subies dans le giron familial, les abus sexuels précoces, l'absence d'instruction et de formation professionnelle et la précarité économique des parents.
\end{abstract}

Mots-clés : Prostitution, jeune fille, maltraitance, précarité économique, violence parentale.

\section{SUMMARY}

The aim of the research was to identify firstly the personal, familial, social and cultural factors which lead underage girls in to prostitution in Bobo-Dioulasso, and secondly, the reasons which led to their situation. A semi-structured interview was administered individually to 15 girls from 14 to 18 years old. Qualitative analysis of the transcript reveals that several risk factors have exposed the girls to prostitution, including: violence and abuse suffered in the family, early sexual abuse, lack of education or vocational training and economic insecurity of parents.

\section{INTRODUCTION}

La prostitution touche tous les continents, même si elle est souvent cachée aux regards, camouflée dans des maisons closes ou protégée par des exploiteurs et des consommateurs de sexe. Au Burkina Faso, la prostitution féminine impliquant des enfants est devenue un phénomène qui prend de plus en plus d'ampleur. Elle inquiète les populations riveraines de certains quartiers populaires où se trouvent de nombreuses chambres de passe. Les autorités municipales sont interpellées par les journaux pour réagir à cette situation qui trouble la tranquillité et la sécurité des populations, y compris des jeunes victimes. Certaines études ont permis de dénombrer 8000 personnes de sexe féminin qui seraient impliquées dans la prostitution à la capitale Ouagadougou (Berthé et Huygens, 2007). Quant à la capitale économique, Bobo-Dioulasso, les femmes en situation de prostitution y seraient environ 3000 , dont des jeunes de 10 à 18 ans (Zio, Saloukou et Mar, 2005). Cette exacerbation de la prostitution des jeunes filles a attiré notre attention à plus d'un titre : d'une part pour comprendre les facteurs qui entraînent des jeunes filles d'âge mineur à se retrouver dans la prostitution et

* Cet article a été élaboré à partir de mon mémoire de maîtrise intitulé La prostitution des jeunes filles mineures à Bobo-Dioulasso au Burkina Faso, Université Laval, Québec, 2011. 
d'autre part pour connaître les caractéristiques sociodémographiques de ces jeunes filles cela afin de mieux prévenir et intervenir. En effet, la prostitution fut longtemps considérée au Burkina Faso comme une pratique exercée majoritairement par des personnes expatriées. Notre préoccupation dans cette recherche est de vérifier également si cette tendance est toujours actuelle particulièrement en ce qui concerne des jeunes filles d'âge mineur.

Les femmes au Burkina Faso représentent 51,7\% de la population, $23 \%$ de celles-ci ont de 10 à 19 ans (INDS, 2006). La majorité de cette jeunesse féminine est sans instruction et sans formation. Cette situation engendre des problèmes sociaux touchant la jeunesse comme la prostitution des mineures.

Certes, la prostitution est une question sensible, particulièrement quand il s'agit des jeunes, car le sujet interpelle nos valeurs et nos repères personnels, sociétaux, culturels et spirituels, ainsi que nos relations à l'argent et aux biens matériels. Pourtant, notre société à travers certains mécanismes injustes (pauvreté, manque de scolarisation, viol, sexualité précoce...) conduit plusieurs jeunes filles à la prostitution.

\section{OBJECTIFS ET MÉTHODE}

Les objectifs de cette recherche étaient :

- d'identifier les caractéristiques personnelles, familiales, sociales et culturelles des jeunes filles mineures de 14 à 18 ans en situation de prostitution à Bobo-Dioulasso;

- d'identifier les facteurs de risque qui ont exposé des jeunes filles mineures à la prostitution.

Pour atteindre nos objectifs, nous avons choisi de mener une recherche de type qualitative qui permettait davantage selon nous, d'explorer et de décrire le phénomène de la prostitution des jeunes filles mineures. Nous avons utilisé un échantillonnage non probabiliste afin d'inclure dans notre recherche les personnes ayant un certain nombre de caractéristiques décrites dans les critères d'inclusion suivants :

- avoir entre 14 et 18 ans;

- $\quad$ être en situation de prostitution depuis au moins un mois à Bobo-Dioulasso;

- $\quad$ se trouver sur les sites sélectionnés pendant la période de la recherche;

- avoir donné son assentiment verbal à la recherche;

- avoir obtenu le consentement d'un parent ou d'un tuteur légal pour participer à la recherche.

Nous avons rencontré dans le cadre de cette étude 15 jeunes filles. Elles ont été rencontrées dans les chambres de passe où elles offrent des services sexuels.

Les entrevues ont duré entre 25 minutes pour la plus courte et 45 minutes pour la plus longue. Les entrevues individuelles avec les participantes ont été réalisées en dioula (langue locale de Bobo-Dioulasso) pour 12 répondantes, en français pour 2 répondantes et en anglais 
pour 1 répondante. Seule 1 répondante n'a pas consenti à l'enregistrement de ses propos. Notre présence sur les sites prostitutionnels dans le cadre de cette recherche a été facilitée par deux associations (Yêrêlon et Association bien-être de la femme - ABF) qui œuvrent auprès de ces jeunes filles dans le cadre de la sensibilisation pour la prévention du VIH/Sida. L'appareil d'enregistrement et le journal de bord ont été les outils qui nous ont permis de faire le traitement de nos données.

La collecte des données s'est faite à travers un guide d'entrevues semi-structuré. Cependant, au fur et à mesure que nous recueillions nos données, nous avons modifié certains mots ou expressions utilisés comme « jeunes filles en situation de prostitution » qui était mieux comprise par les jeunes filles de l'échantillon par le terme dioula «Sunguruba » qui se traduit par «les grandes demoiselles ». Cela leur a permis une meilleure compréhension du sujet au cours des entretiens qui ont suivi. Les sujets suivants ont été abordés dans les entrevues :

- le vécu familial;

- le vécu de la première relation sexuelle;

- la formation scolaire ou professionnelle;

- $\quad$ les motifs d'entrée dans le milieu de la prostitution.

\section{RÉSULTATS}

\section{Caractéristiques sociodémographiques des jeunes filles en situation de prostitution}

Le tableau ci-dessous aide à mieux cerner les caractéristiques sociodémographiques des 15 jeunes filles sujets de cette étude. Chacune d'elle a été désignée par un identifiant anonyme composé de la lettre « $R$ » qui signifie « répondante » et un numéro d'interview accolé. 
Tableau des caractéristiques sociodémographiques des jeunes filles

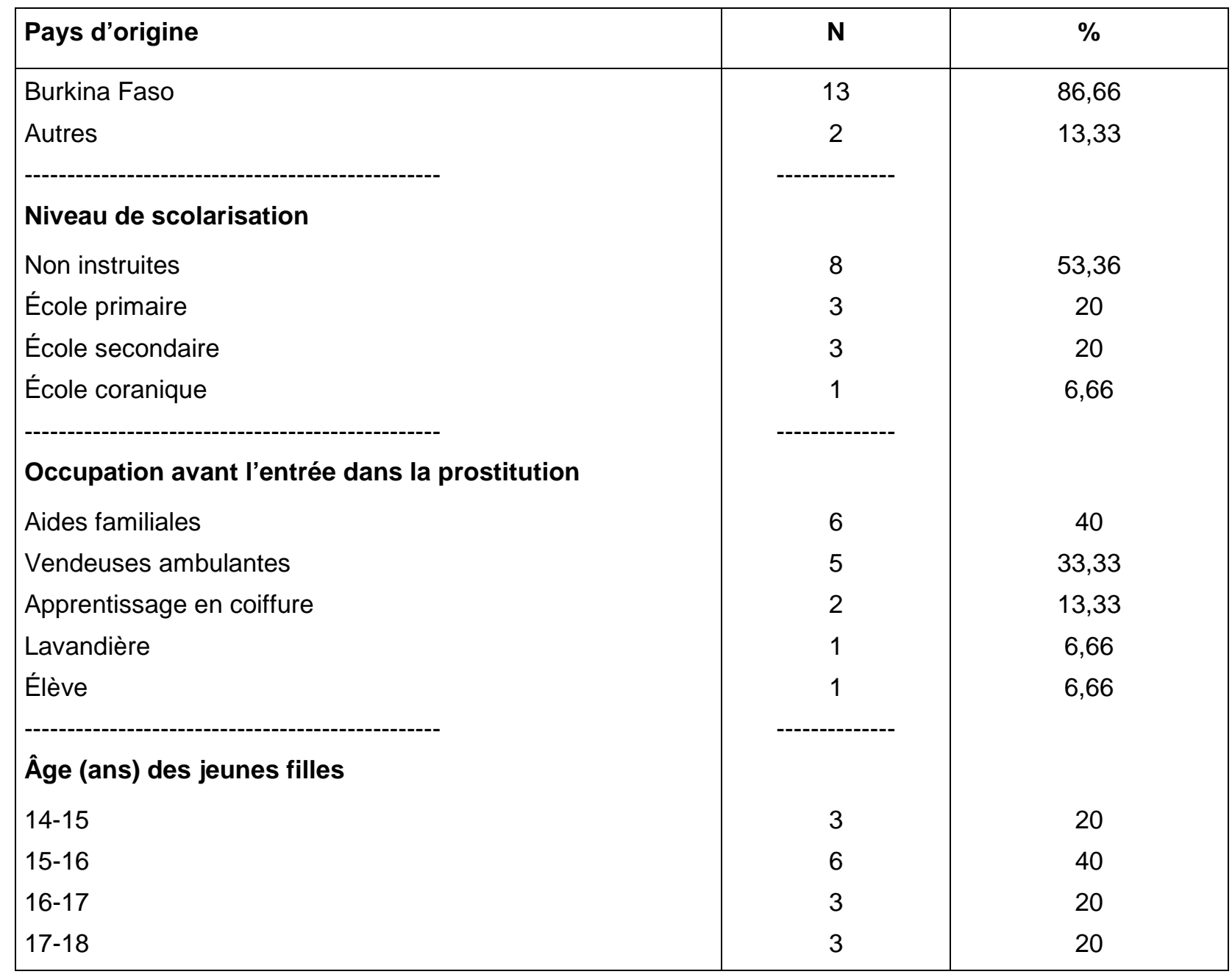

Pays d'origine des jeunes filles

La lecture du tableau nous permet de constater que 13 jeunes filles sont originaires du Burkina Faso et deux proviennent de deux autres pays d'Afrique de l'Ouest: la Côte d'Ivoire et le Nigéria. Bobo-Dioulasso est une ville qui fait le lien entre Ouagadougou et d'autres capitales de la sous-région comme Bamako et Abidjan. Par conséquent, la ville connaît un flux migratoire important. Cependant, le constat est que la majorité des jeunes filles en situation de prostitution rencontrée sont originaires du pays.

Niveau de scolarisation des jeunes filles

Les jeunes filles rencontrées ont des niveaux de scolarisation différents : huit des répondantes n'ont jamais été à l'école; trois ont un niveau primaire; trois ont fréquenté l'école secondaire et une a fait deux années à l'école coranique. 
Je regrette de n'avoir pas eu la chance d'aller à l'école, quelquefois je comprends quand on me parle en français, mais je ne sais pas répondre, j'aimerais aujourd'hui faire l'alphabétisation afin de pouvoir écrire des messages avec mon téléphone portable (R-13).

II faut dire que cette répondante est serveuse dans un bar et donc en contact avec des clients qui généralement parlent français. Cette difficulté de s'exprimer en français à cause de son manque d'instruction crée en elle un complexe et un malaise dans l'exercice de son métier de serveuse.

\section{Occupation des jeunes filles avant leur entrée dans la prostitution}

Le résultat des entrevues révèle que 6 répondantes ont été des aides familiales avant leur entrée dans la prostitution.

Notre échantillon compte une lavandière. Cette jeune fille se promenait avec sa mère de maison en maison pour laver le linge des familles. Cette situation l'a exposée à un abus sexuel à 12 ans avec une grossesse non désirée suivie de son exclusion du domicile familial. Si le manque d'instruction constitue pour certaines jeunes filles un facteur de risque relativement à la prostitution, 1 de nos répondantes élève au secondaire profitait des vacances pour préparer sa prochaine rentrée scolaire à travers la prostitution.

Je viens à Bobo seulement pendant les vacances pour chercher de l'argent et payer mes études de l'année suivante et acheter mes fournitures. L'an passé, j'ai travaillé dans un télécentre. Cette année, ma tante chez qui je viens passer mes vacances m'a demandé de travailler ici dans ce bar, car elle-même travaille dans les chambres de passe parce qu'elle ne pouvait pas me trouver un autre travail. J'ai accepté. Je suis là depuis un mois. Je suis payée à $17500 \mathrm{~F}(43,75$ \$ CAN) et il m'arrive d'avoir des clients généreux qui me donnent plus. Certains me font d'autres propositions que j'accepte aussi, car cela m'aide à augmenter mon gain. Je pourrais ainsi payer aussi la scolarité de mon frère et aider ma mère, car sa situation est pénible au village ( $R-5)$.

On constate que la pauvreté est le facteur principal qui conduit de nombreuses jeunes filles à la prostitution, car leurs parents au village ou en ville sont incapables quelquefois de subvenir à leurs besoins primaires.

\section{Âge des répondantes}

Trois jeunes filles ont entre 14 et 15 ans; six ont entre 15 et 16 ans; trois ont entre 16 et 17 ans et trois ont entre 17 et 18 ans. Notons que la majorité de ces jeunes filles ont commencé leur pratique prostitutionnelle entre 15 et 16 ans. Ce qui correspond à une période où les jeunes filles cherchent à affirmer leur personnalité et à prendre une certaine indépendance vis-à-vis de leurs éducateurs surtout dans des situations où vivent des abus psychologiques, physiques ou sexuels. 


\section{Les facteurs de risque ayant exposé les jeunes filles mineures à la prostitution.}

L'enquête a montré que plusieurs facteurs ont engendré des comportements prostitutionnels chez ces jeunes filles.

\section{Les relations sexuelles précoces}

Plusieurs de ces jeunes filles ont été victimes d'abus sexuels, pour la plupart avant leur entrée dans le milieu prostitutionnel. Une répondante nous a confié avoir eu sa première relation sexuelle parce qu'elle avait faim et cette personne lui promettait $2000 \mathrm{~F}$ (4 \$ CAN).

J'avais 13 ans quand j'ai eu ma première relation sexuelle. C'est le problème d'argent et la faim qui m'ont amenée à avoir ma première expérience sexuelle. II m'a dit qu'il va me donner de l'argent pour acheter des habits et il a couché avec moi et m'a donné 2000F (R-12).

Cette répondante a perdu ses deux parents dû au VIH. Apprenant qu'elle est destinée en mariage à un des fils de sa famille d'accueil, elle a choisi de s'enfuir de son village pour chercher un travail d'aide ménagère à Bobo-Dioulasso alors qu'elle avait 11 ans. Suite au décès de sa patronne, elle se réfugia chez une tante qui lui fait sentir qu'elle était de trop, étant donné ses propres difficultés pour s'occuper de sa progéniture. Cette situation se manifestera par de l'hostilité : même au moment de manger " elle me maltraitait, quand je mangeais elle m'insultait, car je ne donnais rien comme participation ». L'occasion d'exploiter cette situation de faiblesse fut bien saisie par un voisin qui lui proposa de l'argent afin de changer ses guenilles, car elle était toujours très mal vêtue. Il abusa d'elle. Elle se retrouva enceinte et sa tante saisit le prétexte de sa grossesse pour l'exclure définitivement de sa maison.

Deux répondantes nous ont révélé avoir eu leur première relation sexuelle dans une chambre de passe. Cela représente $12 \%$ de l'échantillon. L'une d'elles, la répondante R-4, est orpheline et fut maltraitée dans sa famille d'accueil. Elle fut entraînée par une camarade dans une chambre de passe afin de se procurer rapidement de l'argent pour résoudre ses problèmes financiers et soigner son habillement. Elle fera son initiation dans une chambre de passe. Elle raconte :

Je suis venue ici quand j'avais 15 ans. Je n'avais pas encore eu de relations sexuelles avec un homme. C'est ma copine qui m'a trompée et m'a amenée ici et j'ai commencé cela, car je ne savais rien du tout. Quand je rentrais avec les hommes, je pleurais et criais et le gérant m'a acheté un gel et c'est ainsi que je me suis habituée petit à petit. La première personne qui a eu des relations avec moi ça fait longtemps que je ne l'ai pas vu, il vient ici de temps en temps... II fait partie des clients... Quand je rentrais, les autres filles disaient que je ne connais rien, que je ne connais pas ce travail... (R-4).

On constate que les mauvais traitements infligés à cette orpheline l'ont conduite à un comportement sexuel inadapté qui est la prostitution à 15 ans. Cette répondante est devenue de surcroît la copine du gérant de cette chambre de passe. Ce dernier manifeste son désir de l'épouser, mais n'a pas encore les moyens financiers nécessaires pour cela. 
Une des répondantes a eu sa première relation sexuelle avec un voisin adulte alors qu'elle avait 8 ans. Elle dit en avoir parlé à sa mère, qui a eu des échanges verbaux avec cette personne. Cependant, aucune poursuite judiciaire n'a été envisagée.

\section{La précarité économique}

Les dures conditions économiques que connaissent les familles de ces jeunes filles sont les premiers facteurs de risque qui les conduisirent à entrer dans la prostitution. Ces conditions difficiles de vie sont dues à la paupérisation croissante dans les villes et les campagnes et au chômage chronique.

Certaines coutumes ou traditions sont mises en avant pour se débarrasser des bouches de trop à nourrir, mais les mêmes coutumes deviennent caduques quand les exclus, blâmés hier, reviennent avec de l'argent. C'est cette réalité que ces jeunes filles nous font toucher lorsqu'elles racontent leurs relations avec certains membres de leurs familles:

Ma tante, je lui rends visite de temps en temps. Depuis que je lui donne de l'argent, on s'entend mieux maintenant (R.10). Je suis venue chez une tante ici à Bobo. Mais elle me maltraitait, quand je mangeais elle m'insultait, car je ne donnais rien comme participation. Elle ne me supportait pas (R-12).

\section{La négligence et la maltraitance parentales}

La faiblesse des revenus familiaux et la fragilité des liens avec les parents ont exposé ces jeunes filles à différents types de négligences, de sévérité excessive dans l'éducation. II ressort de notre recherche que 13 jeunes filles sur 15 ont été victimes de négligence, de violence ou de maltraitance avant leur entrée dans la prostitution.

Une dira :

Jusqu'à la fin du monde, je ne vais plus parler à ma tante. C'est à cause d'un problème de chaussure et elle m'a frappée jusqu'à casser sur moi la planche à faire la lessive en bois. Moi je ne veux plus jamais lui adresser la parole (R-11).

Ces différents facteurs ont constitué des motifs d'exclusion de ces jeunes filles de leur milieu social et les ont conduits dans la rue puis dans la prostitution. 


\section{Discussion}

\section{Caractéristiques sociodémographiques des jeunes filles en situation de prostitution}

Nous allons analyser les différentes caractéristiques sociodémographiques des jeunes filles en situation de prostitution rencontrées.

Pays d'origine des jeunes filles

La majorité des jeunes filles d'âge mineur pratiquant la prostitution sont d'origine burkinabè. Cela est compréhensible, car le Burkina Faso n'est pas un pays de forte immigration ${ }^{1}$ à cause de la faiblesse de ses ressources économiques accrues par l'absence de débouché maritime. II faut reconnaître aussi que les frontières sont de plus en plus contrôlées par les autorités policières. L'entrée d'enfants d'autres pays est empêchée par les autorités frontalières. Cependant, Bobo-Dioulasso reste une voie de communication avec la Côte d'Ivoire et le Mali. Le taux de la population immigrée y est plus fort par rapport à la moyenne au pays, mis à part la capitale Ouagadougou. La migration de ces jeunes filles d'origine étrangère, si faible soit-elle, dévoile les difficultés économiques et les problèmes de stabilité politique auxquels font face leurs pays.

\section{Niveau de scolarisation des jeunes filles}

Parmi notre échantillon, 8 jeunes filles sur les 15 rencontrées ne reçurent aucune instruction. Cela reflète le faible taux de scolarisation des jeunes filles malgré les efforts de l'État et des Écoles privées. Les statistiques de 2006 de l'Institut national de la statistique et de la démographie (INDS) révèlent que $73,2 \%$ de la population Burkinabè n'a pas été à l'école. On constate que seulement $21,6 \%$ des femmes sont instruites au Burkina Faso malgré le décret No 285 bis/PRES/EN du 31 août 1965 qui stipule que: «la scolarisation au primaire est obligatoire et gratuite dans les limites des places disponibles ». Le manque de ressources financières amène les familles et l'État à ne pas honorer cet engagement. La non-scolarisation est considérée par certaines jeunes filles interviewées comme un facteur expliquant leur situation de prostitution.

Je n'ai jamais été à l'école et je n'ai bénéficié d'aucune formation. Si j'avais étudié, j'aurais pu devenir riche et ne pas me retrouver ici (R-9).

Cette répondante voit dans l'instruction une protection contre la prostitution parce qu'elle ouvre des opportunités d'emploi. Selon sa vision, l'école est l'une des voies pour avoir des biens matériels et s'enrichir. Une autre répondante constate que son manque d'instruction limite ses possibilités de communication.

1. Recensement général de la Population et de l'Habitat, 2006. 
Occupation des jeunes filles avant leur entrée dans la prostitution

On peut noter une corrélation entre l'occupation des jeunes filles avant leur entrée dans la prostitution et leur niveau de scolarisation. En effet, des jeunes filles sans instruction ni formation n'ont pas de nombreuses options de travail rémunéré, en dehors d'offrir leurs services à des familles - sans parler d'emplois correctement payés. Une de nos répondantes dira :

Auparavant, je travaillais comme bonne dans une famille et j'ai commencé à travailler à 10 ans (R-14).

II faut dire que le travail des enfants n'est pas l'apanage du Burkina Faso. Selon l'Organisation Mondiale du Travail, plus de deux cent cinquante millions d'enfants travaillent à plein temps. L'Afrique compterait $31 \%$ de ces enfants au travail (Ndayisaba et De Grandmont, 1999). Pour certaines de nos répondantes, cette situation est souvent source de plusieurs problèmes pour elles : abus sexuels, avortements, violences physiques, travail sans salaire... Une des répondantes se confiera en ces termes:

Le mari de ma patronne a voulu abuser sexuellement de moi et j'ai quitté mon travail d'aide familiale. Car je le considérais comme un papa. Sa femme était gentille avec moi. Comme il m'était difficile de trouver mieux après, j'ai pris l'offre de travailler dans ce bar. Car je gagne plus ici (R-15).

Au Burkina Faso, une aide familiale gagne entre 3000 et 5000 FCFA (7,50 à 12,50 \$ CAN) par mois. La répondante citée ci-dessus abandonna son travail d'aide familiale pour devenir serveuse de bar, car cela est plus payant. Comme serveuse dans un bar, elle gagne 15000 FCFA, environ 37,50 \$ CAN. On constate que 5 des jeunes filles ont été des vendeuses ambulantes avant leur entrée dans la prostitution. Étant donné les difficultés liées au métier d'aide ménagère, plusieurs jeunes filles non instruites ou qui le sont peu, choisissent aussi le commerce comme occupation pour subvenir à leurs besoins. Une de nos répondantes dira :

J'ai été à l'école jusqu'au CM1, mais j'ai été exclue, car je ne travaillais pas bien. J'ai alors choisi de faire du commerce comme ma mère. Je me promenais comme vendeuse ambulante avec différents articles dans une assiette sur la tête (R-8).

On constate que très peu de possibilités de formation sont accordées à des jeunes filles déscolarisées. Elles secondent souvent leurs mères dans le commerce afin d'augmenter les revenus de la famille ou travaillent pour elles-mêmes. Elles viennent grossir le nombre de jeunes filles n'ayant jamais été à l'école et qui sont exposées à la prostitution. Compte tenu du peu de ressources qu'elles possèdent, elles font le plus souvent du commerce ambulant. On rencontre des jeunes filles sillonnant les rues et les quartiers avec des assiettes de mangues, d'arachides, de salades ou de tomates sur la tête selon la saison. Cette situation les expose à des prédateurs de services sexuels qui n'hésitent pas à leur laisser des pourboires importants, creusant en elles une soif du gain facile. Cela affaiblit leur volonté de se défendre face aux attouchements sexuels de leurs « bienfaiteurs » et les conduits inexorablement à des relations sexuelles tarifiées. Elles découvrent le sexe, mais aussi la possibilité de monnayer les services sexuels pour se faire plus d'argent sans effort. 


\section{Les motifs ayant conduit ces jeunes filles mineures dans le circuit de la prostitution.}

Différentes raisons ont jalonné le parcours de ces jeunes filles pour les conduire à la situation de la prostitution dans laquelle elles se trouvent présentement.

\section{Les relations sexuelles précoces}

Le lien commun entre ces jeunes filles est la précocité de leurs expériences sexuelles. 13 enquêtées sur 15 affirment avoir eu leurs premières relations sexuelles contre leur gré. Une répondante dira :

II m'a trompé pour m'amener dans sa chambre. II m'a forcé et après j'en ai parlé à ma mère et ma mère lui a demandé pourquoi il a fait cela. J'avais autour de 8 ans. Il était notre voisin. (R-13).

Malheureusement au Burkina Faso, plusieurs agressions sexuelles commises sur des mineures sont « arrangées » à l'amiable, c'est-à-dire que la famille de la victime ne dépose pas souvent de plaintes en justice suite à l'agression. D'une part, on cherche à protéger la victime et sa famille afin que celles-ci ne soient pas montrées du doigt par les voisins; d'autre part, on veut souvent éviter que l'accusé aille en prison afin qu'il puisse subvenir aux besoins de sa famille, surtout s'il est le principal pourvoyeur de biens de celle-ci, ou encore pour ne pas nuire à sa réputation. La souffrance, la honte et la culpabilité de la victime sont bien souvent reléguées au second plan. II arrive aussi que des auteurs de viol sur mineures conduits devant les tribunaux écopent d'une peine légère parce qu'on leur aura trouvé des circonstances atténuantes (or la peine prévue au code pour viol sur mineur est de 10 ans d'emprisonnement). Cela à cause des responsabilités que ce dernier a vis-à-vis de sa famille. Malheureusement, ces délinquants sexuels continueront leurs agressions sur d'autres victimes.

Selon l'étude de Tourigny et Laverne (1995), plus la victime abusée sexuellement aura un niveau d'éducation élevé, moins elle serait susceptible de signaler son agresseur. De notre point de vue, cela ne correspond pas du tout à la réalité Burkinabè. De façon générale au Burkina Faso, plus la personne est instruite plus elle fait recours à la justice moins elle est instruite moins elle réclame justice, car l'appareil judiciaire l'intimide même quand elle est dans ses droits.

Une répondante nous a confié avoir eu sa première relation sexuelle avec son oncle quand elle avait 12 ans. Cette situation constitue un inceste dans la mesure où cet oncle fait figure de parent auprès de cette jeune fille. En effet, cette jeune fille ne connaît pas son père. Sa mère dont elle n'a pas de nouvelles sauf qu'elle est dans un des pays de la sous-région ouestafricaine est instable et vit de prostitution. Cette jeune fille a donc été confiée à une sœur de sa mère dont le mari était cet oncle qui avait abusé d'elle, puis l'avait contrainte à avorter. Chargée du commerce de sa tante, elle est accusée de vol d'argent par sa tante. Se disant innocente, elle s'enfuira de la maison pour échapper à la bastonnade et trouvera refuge chez une copine qui est dans le milieu prostitutionnel. 
La précocité des relations sexuelles de ces jeunes filles nous fait penser à cette affiche du «Mouvement Le Cri » dans les travaux de recherches de Judith Trinquart (2002) et qui dit ceci : dans toute prostituée, il y a une petite fille assassinée. Les situations d'abus sexuels conduisent aussi à une dépréciation du corps chez les jeunes filles et à le rendre monnayable.

Les résultats de notre recherche font ainsi écho à l'Étude CAP sur le contexte socioculturel des professionnelles du sexe au Burkina Faso dans le cadre de la lutte contre le VIH/Sida (Zio, Saloukou et Mar, 2005), qui note que 18,2\% des personnes interrogées avaient eu leurs premières relations sexuelles avant 15 ans à Bobo-Dioulasso contre 10,4 \% à Ouagadougou la capitale. On dénombre d'ailleurs un nombre d'agressions sexuelles très élevé chez les jeunes filles à Bobo Dioulasso contrairement aux autres villes du pays. Les médias les relatent, mais plusieurs sont maintenues dans les secrets familiaux.

\section{La précarité économique}

Le Burkina Faso, à l'instar d'autres pays africains, a souffert des conséquences du programme d'ajustement structurel mis en place par la Banque Mondiale et le FMI. L'État a été poussé à brader les services publics. Les institutions publiques comme les écoles, les structures sanitaires et bien d'autres ont été privatisées. Cette situation est mise en exergue par les propriétaires des chambres de passe de Ouagadougou face à la décision du maire de la ville de fermer les chambres de passe.

Nous faisons ce travail parce que nous n'avons pas pu trouver mieux après avoir perdu nos anciens emplois, ayant été victimes des privatisations et certains des crimes économiques. Nous faisons ce travail pour nourrir nos femmes et nos enfants, en refusant la fatalité, pour notre dignité afin de ne pas dépendre d'autrui.

Le libéralisme économique a engendré un coût de vie élevé avec la mise sur le marché de différents biens économiques: téléphone portable, téléviseur, ordinateur, lecteur DVD et bien d'autres, suscitant la convoitise même chez les plus pauvres. Il y a également un monopole des infrastructures dans les villes laissant les villages sans structures de développement économique. II va sans dire que l'indigence a conduit au changement de la solidarité légendaire de certaines familles Burkinabè qui abandonnent leurs valeurs sociales traditionnelles de dignité, de partage, de soutien aux frères et sœurs dans le besoin pour laisser la place à un individualisme croissant.

Dans la situation de précarité économique, les jeunes filles sont les plus touchées parce qu'elles sont femmes et elles ont moins de poids social que les garçons dans une société patriarcale. Lorsqu'elles tombent enceinte avant le mariage, elles sont exclues de la famille temporairement ou définitivement à cause de certains préjugés culturels. Par exemple selon un préjugé culturel, l'accouchement d'une jeune fille dans la cour paternelle provoquerait le décès du père. L'exclusion de la jeune fille cache aussi quelquefois la peur des dépenses liées à une grossesse et à un accouchement surtout quand l'auteur de la grossesse se désengage et laisse la jeune fille seule et face à ses désillusions. Pour Coppieters't Wallant (1990), certaines coutumes par leurs sévérités sont des facteurs conduisant des jeunes filles à la prostitution. La 
peur d'être rejetée par la famille conduit certaines jeunes filles à opter pour un avortement clandestin employant toutes sortes de méthodes et de produits conduisant parfois à la mort. Au Burkina Faso, on a noté en 2009, 23370 avortements dont 1738 cas pratiqués en dehors des structures sanitaires ${ }^{2}$. Plus de la moitié de ces avortements concernerait des adolescentes. Portier-Le Cocq dira (2009:123) :

Le Bureau des recensements de la population mondiale a publié en 1998 les taux de fécondité et de population mondiale. II s'avère que, dans les pays émergents, le taux de maternité adolescente est plus élevé en raison de la pauvreté, du manque d'éducation sexuelle et du fait que les jeunes filles étaient nubiles plus tôt. De plus, le désengagement de la famille et de la communauté en matière de contrôle social est mis en cause.

Pour cet auteur, la grossesse des adolescentes est un signe de l'exclusion sociale, c'est-àdire de la pauvreté qui est un ensemble de problèmes liés au chômage, au manque d'instruction et à la précarité des revenus familiaux.

\section{La négligence et la maltraitance parentales}

Si le nombre de ces mauvais traitements subis par ces jeunes filles (13 sur 15 répondantes) peut paraître préoccupant, le rapport de Tourigny et al. (2002) sur : Étude sur l'incidence et les caractéristiques des situations d'abus, de négligence, d'abandon et de troubles de comportement sérieux signalées à la Direction de la protection de la jeunesse au Québec, témoigne aussi de l'ampleur des mauvais traitements subis par des enfants québécois au sein de leur famille. La différence résiderait dans le taux de signalement plus élevé au Québec à la DPJ qui trouve une alternative de garde pour ces jeunes, alors qu'au Burkina Faso, les signalements à l'Action éducative en milieu ouvert (AEMO) et à la police sont rares et les alternatives sont presque inexistantes. Au Burkina Faso, il n'existe aucune loi interdisant la punition corporelle dans les familles; c'est ce dont témoigne le rapport Burkinabè du comité pour l'élimination et la discrimination à l'égard des femmes dans sa 47e session à l'ONU en ces termes:

Corporal punishment is lawful in the home. Provisions against violence and abuse in Act No.19/61 on juvenile offenders and children at risk, the Penal Code, the Code on the Individual and the Family and the Code of Criminal Procedure are not interpreted as prohibiting corporal punishment in childrearing. ${ }^{3}$

Le même rapport révèle que $83 \%$ des enfants au Burkina Faso de 2 à 14 ans ont fait l'expérience d'une punition corporelle au sein de la famille.

2. Annuaire statistique 2007-2009 DEP Ministère de la Santé

3. Rapport du Burkina Faso sur la discrimination et l'élimination de la violence contre les femmes, lors de la $47^{\mathrm{e}}$ session de l'ONU à Genève en 2010. 


\section{ConClusion}

Notre préoccupation dans cet article a été d'identifier ce qui caractérisait les jeunes filles d'âge mineur en situation de prostitution dans la ville de Bobo-Dioulasso du point de vue de leur origine sociodémographique comme leur niveau de scolarisation, leur occupation avant l'entrée dans le milieu prostitutionnel et leur âge au moment de la recherche.

L'étude nous a révélée que les motifs qui ont conduits les jeunes filles à la situation de prostitution sont: la survenue des relations sexuelles précoces, la précarité économique, la négligence et la maltraitance parentales.

Par notre démarche exploratoire, des jeunes filles ont souvent déploré les abus sexuels subis à l'enfance, la maltraitance dans le giron familial, la précarité économique des parents, l'absence totale ou une insuffisance de scolarisation, le manque de formation professionnelle.

Cette recherche laisse émerger des pistes pour d'éventuelles études ultérieures sur les sujets comme la grossesse chez les jeunes filles en situation de prostitution, l'avortement chez les jeunes filles mineures impliquées dans la prostitution, l'utilisation du préservatif dans la prostitution juvénile.

Yvonne Clémence BAMBARA

Maîtrise en service social (M. Serv.Soc)

BURKINA FASO

\section{BIBLIOGRAPHIE}

Audas, R. et J.D. Willms (2001). Engagement scolaire et décrochage: perspective de la trajectoire de vie. Direction générale de la recherche appliquée, Politique stratégique, développement des ressources humaines du Canada.

Bambara, Y.C. (1999). La réinsertion socio-familiale des jeunes filles marginalisées au Sénégal : l'exemple du foyer Keur Rose Virginie de Dakar, Thèse (M.A.), École nationale des travailleurs sociaux spécialisés, Dakar.

Berthé, A et P. Huygens (2007). «Communiquer avec les femmes vulnérables pour un changement positif de comportement: l'exemple du projet Yêrêlon de Bobo-Dioulasso », Cahiers d'études et de recherche francophones/Santé, vol. 17, $\mathrm{n}^{\circ}{ }^{\circ} 2$, p. 103-109.

Berthé, A. et al. (2008). « Comprendre et atteindre les jeunes travailleuses du sexe clandestines du Burkina Faso pour une meilleure riposte au VIH », Cahiers d'études et de recherche francophones/Santé, vol. 18, $\mathrm{n}^{\circ} 3$, p. 163-173.

Commission sociale des Évêques de France (2001). «L'esclavage de la prostitution », La Documentation catholique, $\mathrm{n}^{\circ}$ 2239, 7 janvier, p. 30-32.

Coppieters't Wallant, R (1990). Jeunesse désœuvrée... Jeunesse d'avenir en Afrique, Bruxelles, Union Catholique Internationale de Service Social. 
Dorais, M. et P. Corriveau (2006). Jeunes filles sous influence, Prostitution juvénile et gangs de rue, Québec, VLB Éditeur.

Dorais, M. et D. Ménard (1987). Les enfants de la prostitution. Québec, VLB Éditeur.

Dorais, M (1993). «Diversité et créativité en recherche qualitative », Service Social, vol. 42, $\mathrm{n}^{\circ} 2$, p. $7-27$.

Lefaso.net (2009). Prostitution des 14 à 17 ans à Ouagadougou.

Ministère de la santé, Burkina Faso, Lutte contre les grossesses non désirées chez les adolescentes et les jeunes: la contraception d'urgence ou pilule du lendemain à la rescousse.

[http://www.lefaso.net/spip.php?page=impression\&id_article=39505] (Consulté 18 novembre 2010).

Ndayisaba, J et N. De Grandmont (1999). Les enfants différents : les comprendre pour mieux les aider, Montréal, Les Éditions Logiques.

O'Deye, A et V. Joseph (2006). La prostitution de mineurs à Paris: Données, acteurs et dispositifs existants. Rapport final, Paris, Cabinet Anthropos.

[http://www.afpssu.com/ressources/la_prostitution_de_mineurs_a_paris__cabinet_anthropos.pdf] (Consulté le 6 janvier 2010).

ONU (2007). Women in Law and Developement in Africa, État des lieux sur les violences faites aux femmes et à la petite fille en Afrique de l'ouest, United nations commission on the status of women.

[http://www.wildaf-ao.org/fr/img/doc/ETAT_DES_LIEUX.doc.] (Consulté le 8 décembre 2009).

ONU (2010). Rapport du Burkina Faso sur la discrimination et l'élimination de la violence contre les femmes, $47^{\mathrm{e}}$ session, Genève.

ONUSIDA (2010). Rapport UNGASS 2010 du Burkina Faso, suivi de la déclaration d'engagement.

[http://www.data.unaids.org/pub/Report/2010/ burkinafaso_2010_country_progress_report_fr.pdf]

(Consulté le 14 novembre 2010).

Portier-Le Cocq, F (2009). Sexualité et maternité des adolescentes: voix anglaises et écossaises, Presse universitaire de Rennes, Collection « Des Sociétés ».

Tourigny, M. et al. (2002). Étude sur l'incidence et les caractéristiques des situations d'abus, de négligence, d'abandon et de troubles de comportement sérieux signalées à la Direction de la protection de la jeunesse au Québec. Rapport final. Montréal, Centre de liaison sur l'intervention et la prévention psychosociales.

Tourigny, M. et C. Lavergne (1995). Les agressions à caractère sexuel. État de la situation, efficacité des programmes de prévention et facteurs associés à la denunciation. Laboratoire de recherche en écologie humaine et sociale université du Québec à Montréal.

Trinquart, J (2002). La décorporalisation dans la pratique prostitutionnelle : un obstacle majeur à l'accès aux soins. Thèse de doctorat.

[http://ecvf.online .fr/IMG/pdf/Trinquart.pdf.] (Consulté le 12 mars 2010). 
UNICEF (2001). Résumé du rapport de l'étude sur les abus sexuels et l'exploitation sexuelle des enfants au Burkina Faso.

[http://www.action-sociale.gov.bf/SiteActionSociale/documents/abus-sexuel.pdf] (Consulté le 12 décembre 2009).

WILDAF/FEDDAL (2007). État des lieux sur les violences faites aux femmes et à la petite fille en Afrique de l'ouest. Commission de la condition de la femme, Organisation des Nations Unies.

[http://www.wildaf-ao.org/fr/img/doc/ETAT_DES_LIEUX.doc.] (Consulté le 8 décembre 2009).

Zio, S.B., L. Saloukou et M.D. Mar (2005). Étude CAP sur le contexte socioculturel des professionnelles de sexe au Burkina Faso dans le cadre de la lutte contre le VIH/Sida. Rapport final. Ouagadougou : Population Council. 